\title{
ARTICLE
}

\section{The Arabidopsis alkaline ceramidase TOD1 is a key turgor pressure regulator in plant cells}

Li-Yu Chen ${ }^{1,2}$, Dong-Qiao Shi ${ }^{1}$, Wen-Juan Zhang ${ }^{1,2}$, Zuo-Shun Tang ${ }^{1}$, Jie Liu ${ }^{1} \&$ Wei-Cai Yang ${ }^{1,3}$

Turgor pressure plays pivotal roles in the growth and movement of walled cells that make up plants and fungi. However, the molecular mechanisms regulating turgor pressure and the coordination between turgor pressure and cell wall remodelling for cell growth remain poorly understood. Here, we report the characterization of Arabidopsis TurgOr regulation Defect 1 (TOD1), which is preferentially expressed in pollen tubes and silique guard cells. We demonstrate that TOD1 is a Golgi-localized alkaline ceramidase. tod1 mutant pollen tubes have higher turgor than wild type and show growth retardation both in pistils and in agarose medium. In addition, tod1 guard cells are insensitive to abscisic acid ( $A B A$ )-induced stomatal closure, whereas sphingosine-1-phosphate, a putative downstream component of $A B A$ signalling and product of alkaline ceramidases, promotes closure in both wild type and tod1. Our data suggest that TOD1 acts in turgor pressure regulation in both guard cells and pollen tubes.

\footnotetext{
${ }^{1}$ State Key Laboratory of Molecular Developmental Biology and National Center for Plant Gene Research (Beijing), Institute of Genetics and Developmental Biology, Chinese Academy of Sciences, Beijing 100101, China. ${ }^{2}$ University of Chinese Academy of Sciences, Beijing 100049, China. ${ }^{3}$ Collaborative Innovation Center for Genetics and Development, Fudan University, Shanghai 200433, China. Correspondence and requests for materials should be addressed to W.C.Y. (email: wcyang@genetics.ac.cn).
} 
A fundamental question in biology is what drives cells to grow and migrate. In animal cells, the cytoskeleton acts as the driving force ${ }^{1,2}$, whereas in the walled cells that make up plants and fungi, turgor pressure is thought to play pivotal roles $^{3-5}$. Cellular turgor pressure is exerted by water moving in response to cellular concentration of ions, sugars and other solutes. Turgor pressure is necessary for cell enlargement, growth and shape maintenance. Pollen tubes and guard cells are two types of highly specialized plant cells, which evolved to allow adaption to life on land ${ }^{6,7}$. In guard cells, turgor pressure is the driving force for stomatal movement. In pollen tubes, turgor pressure is also essential for tip growth. Although turgor pressure is not directly correlated with growth rate, tube growth stops when the turgor pressure is lower than a threshold level ${ }^{8,9}$. Before reaching a plateau, the pollen tube growth rate increases as turgor pressure rises $^{10}$. Furthermore, increased external pressure reduces the growth rate ${ }^{8,10}$. Although these in vitro studies demonstrated that turgor pressure is critical for pollen tube growth, the mechanisms of turgor pressure regulation and the coordination between turgor pressure and the cell wall for in vivo growth, that have attracted plant biologists for a long time are still poorly understood.

In angiosperms, after landing on the stigma, pollen grains hydrate and germinate, and each produces a pollen tube-a single-celled structure that delivers the sperm cells to the ovule ${ }^{11}$. A pollen tube penetrates into the style, and into the transmitting tract, then emerges onto the surface of the septum and grows along the funiculus and is targeted to the micropyle of the ovule ${ }^{12,13}$. The pollen tube finally bursts when it reaches one of the two synergid cells of the ovule, and the two sperm cells are released to enable double fertilization ${ }^{14}$. During this process, pollen tubes need to pass through different tissues of the pistil including papillar cells of the stigma, the transmitting tract, the septum and the filiform apparatus ${ }^{13}$. To penetrate these 'physical barriers' of the pistil, pollen tubes must regulate their turgor pressure during in vivo growth.

Sphingolipids are ubiquitously present in all eukaryotic cells and in a few bacteria ${ }^{15}$. They are thought to be essential for membrane structure and are also involved in signal transduction, controlling essential cellular processes such as apoptosis, cell migration, differentiation and inflammation ${ }^{15}$. Ceramide, sphingosine, sphingosine-1-phosphate (S1P) and their derivatives are examples of a class of well-studied bioactive sphingolipids in animals. Ceramidases hydrolyse ceramide to yield sphingosine and fatty acid. They play a key role in sphingolipid metabolism, being responsible for regulatory activity at the cellular level. Ceramidases are classified as acidic, neutral or alkaline, based on their optimal $\mathrm{pH}$ for biological activity. Based on sequence homology, the Arabidopsis genome encodes one alkaline phytoceramidase ${ }^{16}$ and three neutral ceramidases ${ }^{17}$, but their functions have not been characterized.

Here, we show that TurgOr regulation Defect 1 (TOD1) is a bona fide alkaline ceramidase that is preferentially expressed in pollen tubes and in silique guard cells, where it is required for turgor pressure regulation. Disruption of TOD1 results in partial male sterility, which is due to defects of pollen tube growth in penetrating the pistils. tod 1 mutant pollen tubes also show growth retardation inside agarose medium; this retardation is rescued by application of sphingosine or S1P. These data suggest that maintenance of turgor pressure likely controlled by TOD1 is needed for pollen tube growth in pistil. Furthermore, our work provides novel insights into the role of sphingolipid signalling pathways during pollen tube growth in plants.

\section{Results}

Isolation of the tod1 mutant. We screened gene trap lines of Arabidopsis thaliana ecotype Landsberg erecta ${ }^{18}$ for mutants defective in pollen tube growth in vivo and thereby identified one mutant, designated TurgOr regulation Defect 1 (tod1). Mutant plants bore short siliques resulting from severe sterility (Fig. 1a). The average silique length of homozygous tod 1 was about one-half that of the wild-type (Fig. 1b) and only about $18 \%$ of the available ovules were fertilized to produce seeds (Fig. 1c). The $F_{1}$ progeny of the heterozygous mutant showed a 1:1 $\left(271: 265, \chi^{2}<\chi_{0.01}^{2}\right)$ segregation ratio of kanamycin-resistant $\left(\operatorname{Kan}^{\mathrm{R}}\right)$ to kanamycin-sensitive $\left(\operatorname{Kan}^{\mathrm{S}}\right)$, indicating that the $D s$ insertion affect the function of either the male or female gametophyte $^{19}$. To determine which sex was affected, we conducted reciprocal crosses. When wild type was used as a pollen donor on heterozygous tod 1 flower, the segregation ratio of the $F_{1}$ progeny was $0.94: 1 \quad(191: 203)$, indicating that the kanamycin-resistant gene NPTII was transmitted by the female gametophyte. However, when pollen from heterozygous tod 1 was used to pollinate wild-type flowers, the segregation ratio of $\mathrm{Kan}^{\mathrm{R}}: \mathrm{Kan}^{\mathrm{S}}$ in the $\mathrm{F}_{1}$ progeny was $0.025: 1$ (15:601), indicating that NPTII was barely be transmitted by the male gametophyte. These data confirmed that the mutation affects the male gametophyte.

tod1 is defective in in vivo pollen tube growth. To investigate the cause of male sterility, mature pollen grains were stained with Alexander's stain and with 4',6-diamidino-2-phenylindole (DAPI) and analysed by microscopy. The cytoplasm of tod 1 pollen was red purple after Alexander's staining (Supplementary Fig. 1a), indicating that the viability of the mutant pollen grains was not affected ${ }^{20}$. DAPI staining showed two bright sperm nuclei and one more diffuse vegetative nucleus, as found in wildtype plants (Supplementary Fig. 1b). To determine whether the mutation caused defects in pollen germination and/or pollen tube growth, we checked in vitro pollen germination rates and tube growth. Both wild-type and the mutant pollen yielded above $75 \%$ germination after $5 \mathrm{~h}$ incubation (Supplementary Fig. 1c). There were also no differences between in vitro pollen tube length of wild type and mutant after $5 \mathrm{~h}$ germination (Supplementary Fig. 1d).

To further check whether tod 1 had defects in in vivo pollen tube growth, wild-type and mutant pollen tubes growing in Ler pistils were stained with aniline blue. tod 1 pollen tubes showed reduced growth potential (Supplementary Fig. 2), and only a few pollen tubes had reached the middle of the pistil $24 \mathrm{~h}$ after pollination, whereas wild-type pollen tubes had reached the base of the pistil (Fig. 1d and Supplementary Fig. 2). We also checked the status of pollen tubes $48 \mathrm{~h}$ after pollination, when almost all ovules were fertilized and pollen tubes began to degenerate in the wild type (Supplementary Fig. 3); most tod1 pollen tubes were still in the top half of the pistil, and many of them had begun to degenerate (Supplementary Fig. 3). Consistent with the in vivo phenotype of pollen tubes, the seeds distribution in tod 1 siliques was distorted, with more seeds at the stigma end (Fig. 1e). It seemed that the mutant pollen tubes had lost the ability to penetrate efficiently through the style.

To confirm whether the low seed set phenotype was also caused by defects in pollen tube guidance in the gametophytic phase, we observed tod1 pollen tubes in detail by aniline blue staining and scanning electron microscopy (SEM). Once the mutant pollen tubes penetrated the septum walls, they grew along the funiculus and then entered the micropyle of the ovule to complete fertilization as seen for wild-type tubes (Supplementary Fig. 4a-d). Limited pollinations showed that only about $10 \%$ of tod1 pollen tubes in the transmitting tract could go through the septum wall; more than $95 \%$ of septum-penetrating tod 1 pollen tubes could enter the micropyle of the ovule 
a
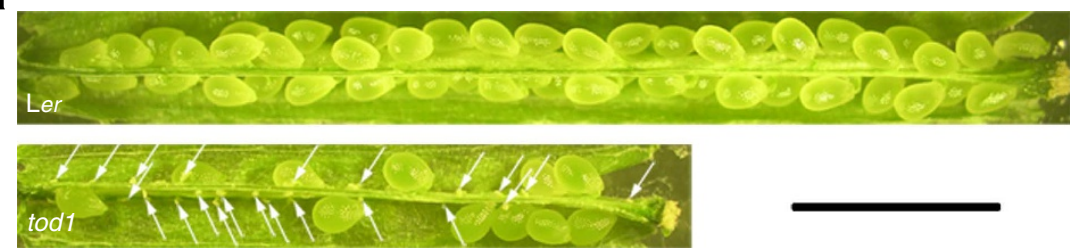

b

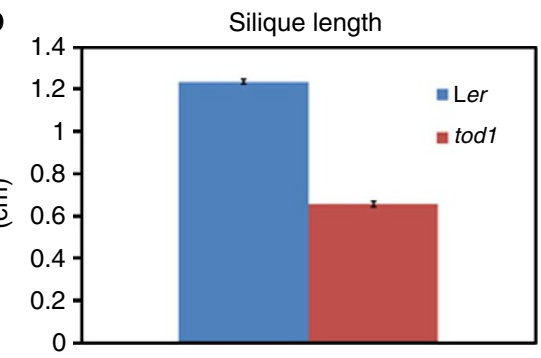

C

Normal seed ratio

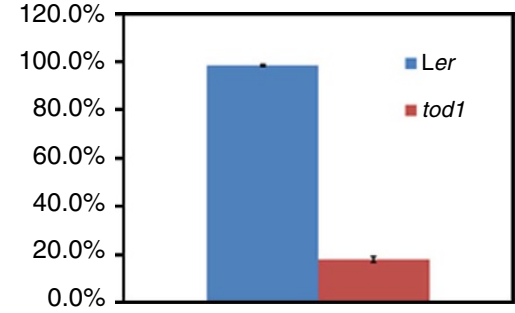

d
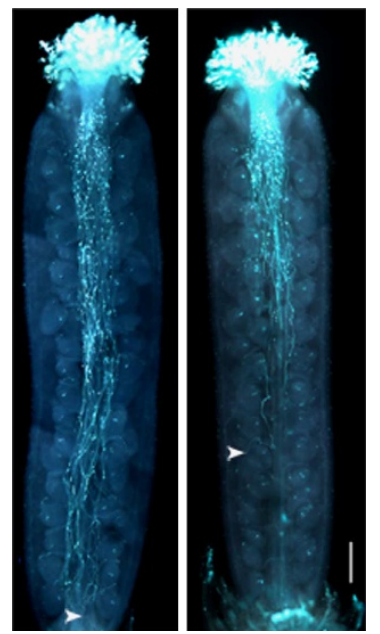

e
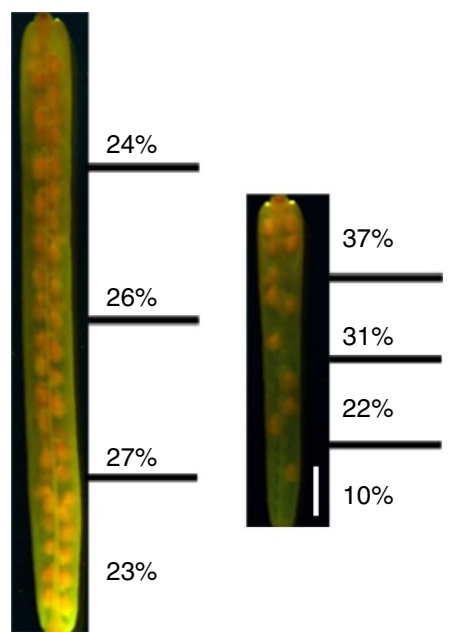

Figure 1 | Phenotype of tod1 mutant. (a) Dissected siliques from Ler and tod1. Arrows point to aborted ovules. Scale bar, $2 \mathrm{~mm}$. (b) Silique lengths of Ler and tod1. Data are mean \pm s.e. $(n=72)$. (c) Seed ratios of Ler and tod1. Data are mean \pm s.e. $(n=33)$. (d) tod1 pollen tubes showed growth defects in vivo. Ler pollen tubes (left panel) and tod7 pollen tubes (right panel) in Ler pistils stained with aniline blue $24 \mathrm{~h}$ after pollination. The longest pollen tubes are indicated by arrowheads. Scale bar, $200 \mu \mathrm{m}$. (e) Seed distribution in sliques from Ler (left panel) and tod1 (right panel) selfed plants. Siliques were cleared by ethanol and images were recorded using a stereo microscope equipped with CCD (Ler, $n=42 ;$ tod1, $n=43$ ). Scale bar, $1 \mathrm{~mm}$.

(Supplementary Fig. 4e). This suggests that pollen tube guidance in the gametophytic phase was not affected in the mutant.

Molecular cloning of TOD1. We performed thermal asymmetric interlaced $\mathrm{PCR}^{21}$ to obtain the flanking genomic sequence of the $D s$ element. Sequencing indicated that the $D s$ element was inserted into the first exon of At5g46220, 114-bp downstream of the ATG (Fig. 2a). The Ds insertion resulted in an 8-bp duplication $\left(5^{\prime}\right.$-CTCTCAAG- $\left.3^{\prime}\right)$ at the insertion site. Furthermore, we were unable to detect TOD1 transcripts in inflorescence RNA, indicating that tod 1 was a null allele (Fig. $2 \mathrm{~b}$ ).

To confirm that the Ds insertion in TOD1 was indeed the cause of the mutant phenotype, we transformed a 5,557-bp genomic DNA fragment of TOD1, containing the predicted promoter region, open reading frame and $3^{\prime}$-untranscribed region ( $3^{\prime}$-UTR) into homozygous tod 1 plants. Eighty-seven independent T1 transgenic lines were obtained. We randomly selected ten lines for analysis of silique length and seed set. Fertility was restored in complemented mutant plants (Supplementary Table 1), indicating that TOD1 was sufficient to rescue pollen tube growth defect. In addition, SALK_110396, which harboured a T-DNA insertion in the first exon of At5g46220 (Fig. 2a), named tod1-2, also displayed the similar phenotype as observed in tod1-1.

TOD1 is predicted to encode a 462 -amino-acid protein, with a transmembrane domain (residues 20-39), and a DUF616 domain (residues 107-409; Fig. 2c), but no other homology to proteins with known functions. However, the PANTHER (Protein ANalysis THrough Evolutionary Relationships) analysis classified TOD1 as a member of a family of alkaline ceramidases, although they have no similarity at the amino-acid level (http:// www.pantherdb.org/ $)^{22,23}$. We generated a phylogenetic tree using TOD1 orthologues from other plant species and other eukaryotic organisms, all of which belong to the alkaline ceramidase family. Interestingly, these members clustered into two clades: one contains plant-specific proteins and the other includes proteins belonging to other eukaryotic organisms (Supplementary Fig. 5a). The plant-specific clade, including TOD1 from Arabidopsis, contains members from eudicots including grape (Vitis vinifera), soybean (Glycine max), Medicago truncatula, poplar (Populus trichocarpa) and monocots such as rice (Oryza sativa). These members' functions are currently not known. The other clade contains 
a

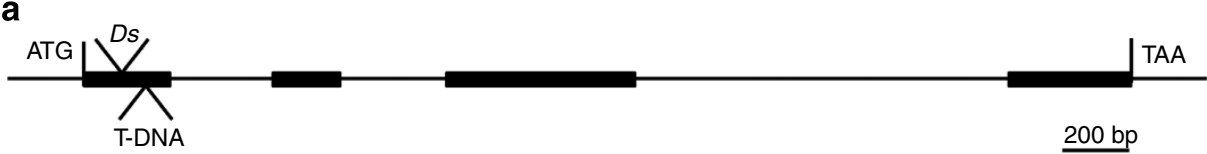

b

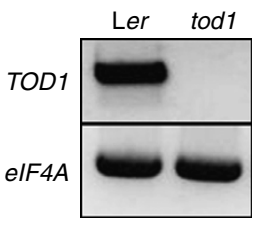

d

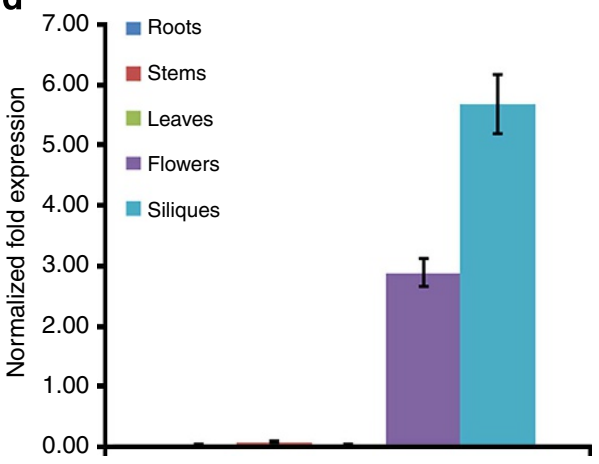

C

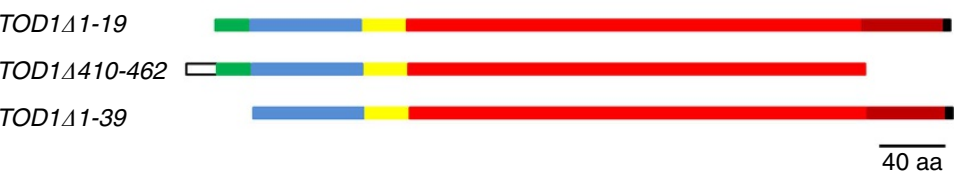

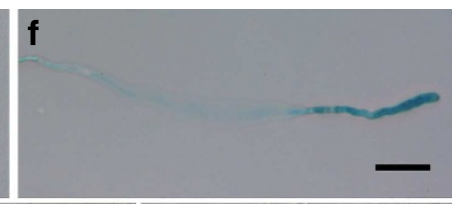
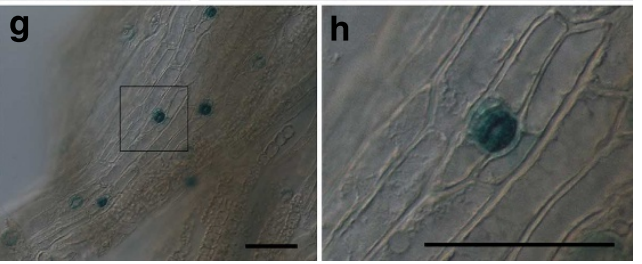

Figure 2 | Detection of mutations causing partial male sterility in tod1 mutants and expression pattern of TOD1. (a) Genomic structure of TOD1. Ds and T-DNA inserted into the first exon at 114-bp and 186-bp downstream of start codon in tod1-1 and tod1-2, respectively. (b) Full-length TOD1 transcripts are absent in the mutant. Total RNA from inflorescences was reverse-transcribed and amplified by PCR. (c) Scheme of TOD1. It contains a transmembrane domain at the $\mathrm{N}$ terminus (residues 20-39), and a DUF616 domain (residues 107-409). Amino acids 133-458 are annotated as an alkaline phytoceramidase domain by PANTHER. (d) Real-time RT-PCR of TOD1 expression pattern in different tissues of Arabidopsis. Data are mean \pm s.e. $(n=4)$. (e-h) Cell-specific pTOD1:::gTOD1-GUS expression. (e) Mature pollen grain, (f) pollen tube, ( $(\mathbf{g})$ guard cells on a silique and (h) magnification of the area indicated in $\mathbf{g}$. Scale bars, $50 \mu \mathrm{m}$.
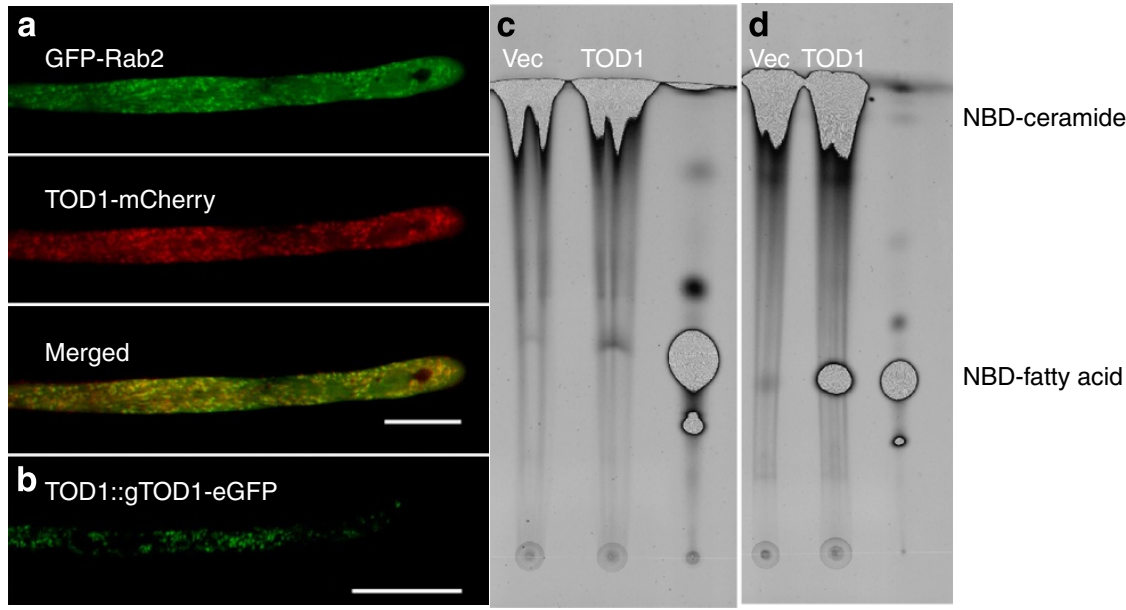

Figure 3 | TOD1 is a bona fide alkaline ceramidase localized in the Golgi apparatus. (a) CLSM images showing that TOD1-mCherry co-localizes with Golgi marker GFP-Rab2. pLat52::TOD1-mCherry and pLat52::GFP-Rab2 were co-transfected and transiently expressed in tobacco pollen tubes. Scale bar, $20 \mu \mathrm{m}$. (b) TOD1-eGFP localization in an Arabidopsis pollen tube from plants stably transformed with pTOD1::gTOD1-eGFP. Scale bar, $20 \mu \mathrm{m}$. (c,d) TOD1 has alkaline ceramidase activity. Expression of TOD1 was induced in yeast mutant $\Delta y p c 1 \Delta y d c 1$, which lacks two endogenous yeast ceramidases. Total microsomes from $\Delta y p c 1 \Delta y d c 1$ containing empty vector pYes2 (Vec) or pYes2-Flag-TOD1 (TOD1) incubated with the fluorescent substrate NBD-ceramide. The third lane is the NBD-fatty acid standard. (c) $\mathrm{pH}$ 7.0. (d) $\mathrm{pH} 9.4$.

members from yeast (Saccharomyces cerevisiae), human (Homo sapiens) and mouse (Mus musculus). Their functions have been characterized as alkaline ceramidases ${ }^{24-26}$, alkaline phytoceramidases $^{16,27}$ or alkaline dihydroceramidases ${ }^{28,29}$. The sequence similarity between these two clades is very low, but they share high similarity within their clades (Supplementary Fig. 5b).
The region comprising amino acids $133-458$ is predicted as a putative alkaline phytoceramidase domain (Fig. 2c). To test the function of the gene, we constructed three deletion variants of TOD1 to complement the mutant phenotype (Fig. 2c). Only TOD1 11-19, which contains the transmembrane domain and the putative alkaline phytoceramidase domain, could restore the seed 
set of the mutant. Neither TOD1 $4410-462$ nor TOD1 1-39 could complement the mutant phenotype. These results indicate that both the transmembrane domain and putative phytoceramidase domain are critical for TOD1 function.

TOD1 is expressed in pollen and silique guard cells. Real-time reverse transcription (RT)-PCR showed that TOD1 transcripts were present in flowers and siliques, were barely detectable in roots, stems or leaves (Fig. 2d). To further investigate the cellular expression pattern of TOD1, we examined pTOD1::gTOD1-GUS transgenic lines which express $\beta$-glucuronidase (GUS); TOD1 was preferentially expressed in pollen grains, pollen tubes and silique guard cells (Fig. 2e-h). To characterize the subcellular localization of TOD1, we co-transformed pLat52::TOD1-mCherry with endomembrane system markers into tobacco (Nicotiana tabacum) pollen grains by bombardment. Confocal laser scanning microscopy (CLSM) showed that TOD1 co-localized only with Golgi marker GFP-Rab2 (Fig. 3a). Moreover, pollen tubes from transgenic Arabidopsis plants expressing TOD1-enhanced green fluorescent protein (eGFP) under its native promoter also showed similar localization pattern (Fig. 3b). Thus, TOD1 is a Golgilocalized protein in pollen tubes.

TOD1 is a bona fide alkaline ceramidase. To test whether TOD1 has ceramidase activity, we inserted the full-length TOD1-coding sequence with a FLAG tag at its amino terminus into the yeast expression vector pYES2, under the control of the GAL1 promoter. The resulting construct was transformed into the yeast mutant $\Delta y p c 1 \Delta y d c 1$, lacking two endogenous yeast ceramidases YPC1p and YDC1 ${ }^{28}$. The expression of Flag-tagged TOD1 was induced by galactose and confirmed by western blot analysis using an anti-Flag antibody (Supplementary Fig. 6a). Total microsomal extracts were prepared from yeast cells and used for enzyme activity assays with $\mathrm{NBD}-\mathrm{C}_{12}$-phytoceramide (with the $\mathrm{C}-4$ hydroxyl group) or NBD-C 12 -ceramide (with the $\Delta 4$-double bond) as substrate. The thin-layer chromatography (TLC) showed that phytoceramide is not a substrate for TOD1 (Supplementary Fig. 6b), but ceramide is (Fig 3c,d). Furthermore, TOD1 displayed stronger ceramidase activity at pH 9.5 than at pH 7.0 (Fig. 3c,d). Taken together, we conclude that TOD1 is an alkaline ceramidase.

TOD1 regulates turgor in guard cells and pollen tubes. As $T O D 1$ is expressed both in pollen tubes and in silique guard cells, we checked whether the stomata on siliques had defects in response to abscisic acid (ABA) or S1P. Stomata on siliques had similar response to $\mathrm{ABA}$ as stomata on leaves (Fig. 4a). ABA at concentration of $10 \mu \mathrm{M}$ promotes pre-opened stomata closing in Ler, but not in $\operatorname{tod} 1$ (Fig. 4a), indicating that $\operatorname{tod} 1$ is insensitive to exogenous ABA. ABA can activate sphingosine kinases (SphKs), which will phosphorylate sphingosine, the product of the ceramidase reaction, and subsequently increase S1P level ${ }^{30}$. S1P is known to play a positive role in stomatal closure ${ }^{31}$. Interestingly, exogenous S1P $(10 \mu \mathrm{M})$ promoted closure of pre-opened stomata both in Ler and tod1 (Fig. 4b). These data suggested that TOD1 is also involved in ABA-induced guard cell turgor regulation, likely acting downstream of $\mathrm{ABA}$ and upstream of S1P.

To confirm whether the in vivo growth defects of tod 1 pollen tubes could be caused by altered turgor pressure, we germinated pollen grains inside germination medium supplemented with $1.5 \%$ agarose. After $10 \mathrm{~h}$ growth, the average pollen tube length of tod1 pollen was about $130 \mu \mathrm{m}$, half of the wild-type length (Fig. 4c). As there was no difference in pollen tube growth between wild-type and tod 1 on agarose medium as shown earlier (Supplementary Fig. 1d), it seemed possible that the mutant pollen tubes were defective in turgor pressure regulation, thus leading to the reduced penetration potential. To further check whether sphingosine or S1P could rescue the growth defects of tod 1 pollen tubes inside the germination medium with $1.5 \%$ agarose, we measured the average length of mutant pollen tubes germinated in the presence of $10 \mu \mathrm{M}$ sphingosine or S1P. The average length of mutant pollen tubes increased from $130 \mu \mathrm{m}$ (without) to about $245 \mu \mathrm{m}$ (with sphingosine or S1P; Fig. 4c), indicating that both sphingosine and S1P can rescue the growth defects of mutant pollen tubes with higher external pressure.

To test whether the tod 1 mutation affected turgor pressure, we tried to measure pollen tube turgor pressure by the pressure probe and incipient plasmolysis methods. However, Arabidopsis pollen tubes are too small for the pressure probe, so instead we used the incipient plasmolysis method for turgor pressure measurement. The tod 1 pollen tubes generated higher turgor pressure than wild-type pollen tubes (Fig. 4d). Therefore, we conclude that a mutation in the TOD1 gene results in disregulation of turgor pressure.

As S1P is involved in the regulation of $\left[\mathrm{Ca}^{2+}\right]_{c y t}$ in animal cells and guard cells, we also examined $\left[\mathrm{Ca}^{2+}\right]_{c y t}$ in growing pollen tubes with a YC3.60 cameleon probe. The Venus/ECFP ratio imaging showed that the tod 1 pollen tubes had decreased $\left[\mathrm{Ca}^{2+}\right]_{\text {cyt }}$ (Fig. 4e).

To provide further genetic evidence for TOD1's role in turgor regulation, we screened for extragenic suppressors of the tod 1 mutation and isolated one suppressor that rescued the reduced seed set defect of tod1. Whole-genome re-sequencing and SNP (single-nucleotide polymorphism) analysis results revealed that the suppressor mutant had a single-nucleotide substitution in the Galacturonosyltransferase13 (GAUT13) gene, resulting in a premature stop codon. GAUT13 and its homologue GAUT14 function redundantly in pollen tube growth, possibly through pectin biosynthesis of the pollen tube wall ${ }^{32}$. Pollen tubes of the gaut13 gaut14 double mutant are swollen and defective in elongation, both in in vitro and in vivo ${ }^{32}$. To confirm the re-sequencing result, we crossed two T-DNA insertion lines of tod1-2 and gaut13-1, and obtained homozygous double mutants in the $\mathrm{F}_{2}$ generation. The pollen tube growth defects of tod $1-2$ were indeed suppressed by the gaut 13 mutation (Fig. $4 \mathrm{f}$ ). Double mutant pollen tubes arrived at the base of the pistil $12 \mathrm{~h}$ after pollination, and grew through the septum wall and target the micropyle of the ovule (Fig. 4f). The seed ratio of double mutant plants was restored from the $23 \%$ seen with the tod1-2 single mutant to $89 \%$ (Supplementary Fig. 7).

\section{Discussion}

To date, the role of turgor pressure in pollen tube growth is controversial ${ }^{33-36}$. Here, we identified a $D s$ insertion mutant, tod1, in which in vivo pollen tube growth was defective. Disruption of TOD1 caused partial male sterility, resulting from the reduced growth potential of mutant pollen tubes. Detailed examination indicated that the ability of todl pollen tubes to penetrate pistil tissues was severely compromised. tod1 pollen tubes also showed growth defects inside medium solidified with $1.5 \%$ agarose, in which the external pressure was higher than normal conditions. We attributed this inability to disregulation of turgor pressure of the mutant pollen tubes.

Pollen tube growth is regulated by the coordination of turgor pressure and cell wall stiffness. Turgor pressure is thought to be the driving force, whereas the cell wall is thought to be the speed setter. For example, application of high concentrations of pectinase caused apical swelling or bursting of pollen tubes, whereas application of moderate concentration pectinase stimulated tube growth ${ }^{37}$. It was therefore hypothesized that pectinase 

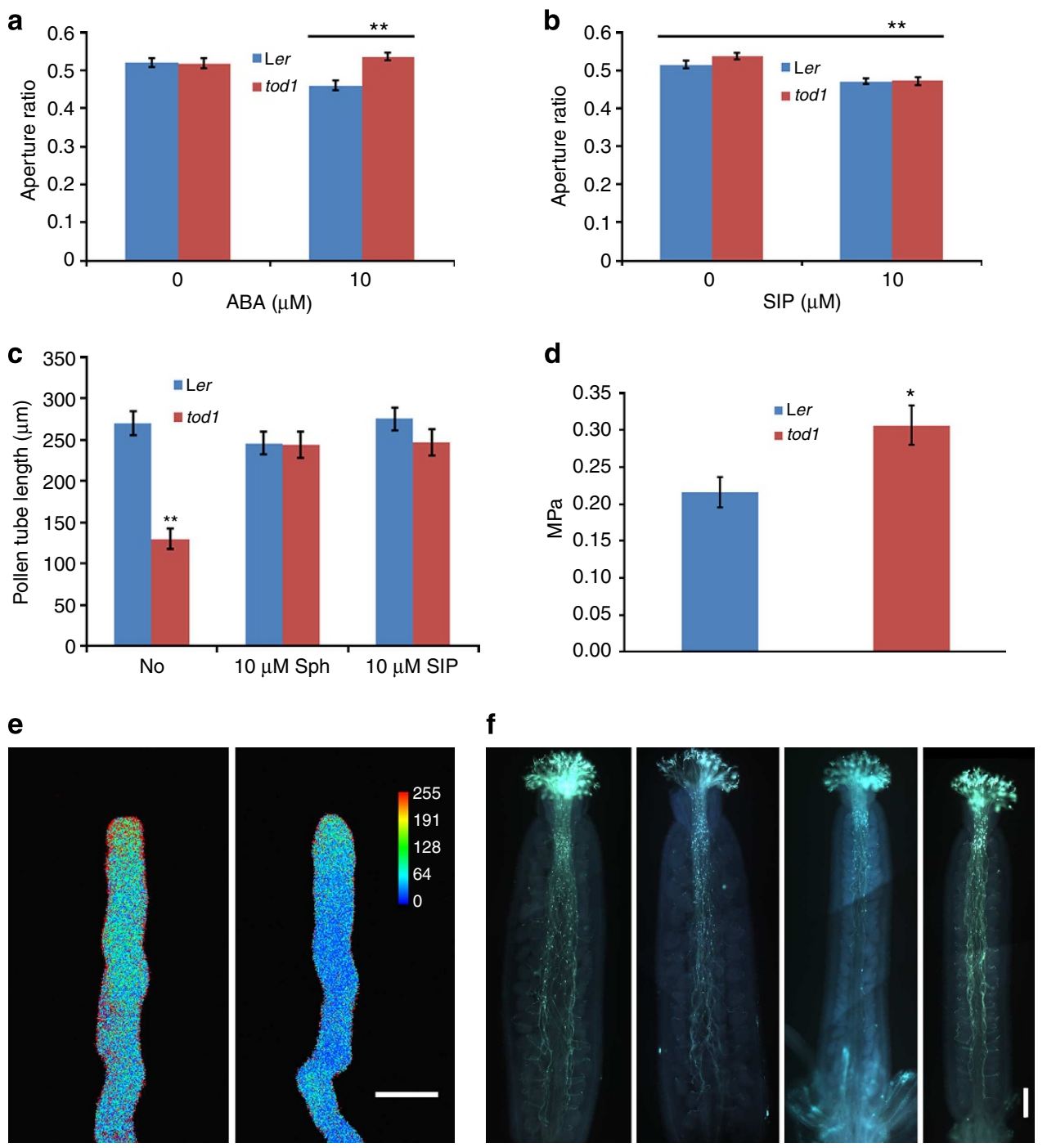

Figure 4 | tod1 mutants have a turgor problem. (a) Exogenous $10 \mu \mathrm{M}$ ABA promotes pre-opened stomata closure in Ler, but not in tod1. Data are mean (width/length) \pm s.e. $(n=48)$. (b) Exogenous $10 \mu \mathrm{M}$ S1P promotes closure of pre-opened stomata both in Ler and tod1. Data are mean (width/length) \pm s.e. $(n=48)$. (c) tod1 pollen tubes grow slower than those of wild type inside pollen tube germination medium with $1.5 \%$ agarose. Growth defects of mutant pollen tubes can be rescued by adding $10 \mu \mathrm{M}$ sphingosine (Sph) or S1P. Data are mean \pm s.e. $(n>55)$. (d) tod1 pollen tubes generate a higher turgor pressure than wild-type pollen tubes. Data are mean \pm s.e. (Ler, $n=19 ;$ tod1, $n=10$ ). (e) Typical YC3.60 cameleon Venus/ECFP ratio images of maximum $\left[\mathrm{Ca}^{2+}\right]_{\text {cyt }}$ in growing pollen tubes. Left panel, Col pollen tube expressing YC3.60. Right panel, tod1-2 pollen tube expressing YC3.60. The coloured bar represents the relative cytosolic $\mathrm{Ca}^{2}+$ levels. Scale bar, $10 \mu \mathrm{m}$. (f) Suppression of tod1-2 pollen tube growth defect by gaut13-1 mutation. Col, gaut13-1, tod1-2 and tod1-2 gaut13-1 double mutant pollen tubes in Col pistils (from left to right) stained with aniline blue $12 \mathrm{~h}$ after pollination. Scale bar, $200 \mu \mathrm{m}$. Student's t-test, ${ }^{\star} P<0.05 ;{ }^{\star \star} P<0.01$.

that digest pectins, the main cell wall component of pollen tubes, sets the tube growth rate by regulating the physical characteristics of the tube cell wall. Silencing of the gene encoding tobacco pollen pectin methylesterase involved in the building up of the pollen tube wall strength, resulted in retarded in vivo pollen tube growth, whereas in vitro pollen tube growth was not affected ${ }^{38}$. This suggests that the stiffness of pollen tube wall should be kept at an optimum. Turgor pressure also needs to be maintained at an optimal and in a narrow range, neither too high nor too low, although the growth rate is likely not correlated with turgor pressure ${ }^{8}$. tod 1 pollen tubes generate higher turgor pressure than the wild-type ones as measured by the incipient plasmolysis method (Fig. 4d). Much higher turgor pressure is expected during in vivo growth since pollen tubes need to resist external pressure from pistil tissues during their penetration. Why are mutant tubes with higher turgor pressure defective in in vivo growth? One explanation is that the turgor pressure of the mutant tubes, exceeding the in vivo pressure optimal for wild-type tubes, impairs tube growth. In addition, the increased turgor pressure in tod1 pollen tubes may also affect the building up of the pollen tube wall strength, which might also cause in vivo retarded growth. To resist the increased turgor pressure from bursting, tod1 mutant pollen tubes might deposit much more wall material, which in turn would display reduced growth potential. Finding that gaut 13 acts as a suppressor of tod 1 supports this hypothesis, because mutation of GAUT13 has quantitative effects on cell wall deposition.

Sphingolipids are ubiquitous in eukaryotes and some bacteria. The biosynthesis and metabolism of sphingolipids have been well studied for the last two decades. Ceramide is mainly synthesized in the ER (endoplasmic reticulum), and is then transported to the Golgi apparatus for further modifications. TOD1 shares low sequence similarity with reported alkaline ceramidases from human $^{25}$ and mouse ${ }^{24}$. We showed here that TOD1 possesses 
ceramidase activity optimally at alkaline $\mathrm{pH}$ and it is localized to the Golgi apparatus. Human alkaline ceramidase 2 (haCER2), which shows a $\mathrm{pH}$ optimum of 8.0, is also localized to the Golgi apparatus $^{25}$. In eukaryotic cells, different organelles exhibit variable $\mathrm{pH}$ to fulfill different requirements for protein function within them. Although the average $\mathrm{pH}$ value of the Golgi is about 6.4 (ref. 39), it can be alkalinized by $\mathrm{Na}^{+} / \mathrm{H}^{+}$exchangers on its membrane ${ }^{40}$. It is possible that rapid activation of these ion exchangers might generate highly alkalinized area in close proximity to Golgi membranes. Furthermore, TOD1 also had weak ceramidase activity at $\mathrm{pH} 7.0$, so it is likely that a basal level of activity is present in the Golgi at neutral $\mathrm{pH}$. The possible coupling between TOD1 activity and the activation of ion exchangers in Golgi membrane is of special interest for further investigation.

In animal cells, ceramide, sphingosine, S1P and their derivatives are attracting considerable interest because of their roles in intra- and extracellular signalling. However, recent studies using lipidomic analyses suggested that the abundance of $\Delta 4$-unsaturated sphingolipdids is low in Arabidopsis ${ }^{41,42}$. This is not surprising that, because work from Michaelson and colleagues $^{42}$ and our data, several key enzymes involved in metabolism of $\Delta 4$-unsaturated sphingolipdids metabolism are restrictedly expressed in limited cell types. Therefore, sphingolipds signalling may function only in specific plant cells. S1P had been proposed to act positively in stomatal closure from pharmacological experiments ${ }^{30}$. Stomatal activity is also regulated by turgor pressure. Our results indicate that stomatal closure is defective in guard cells of tod1 siliques, and this can be rescued by exogenous application of S1P, confirming that TOD1 acts upstream of S1P in stomatal closure. In pollen tubes, disruption of TOD1 also results in higher turgor pressure, and the pollen tube growth retardation caused by increased external pressure can be rescued by exogenous application of S1P. These findings suggest that TOD1 acts similarly both in guard cells and pollen tubes, where it is preferentially expressed.

Based on sequence homology, six SphKs were identified in the Arabidopsis genome ${ }^{43,44}$, three of which LCBK1 (ref. 45), SphK1 (ref. 43) and SphK2 (ref. 44), were confirmed to have kinase activity towards sphingosine. As these three $S p h K$ genes are all expressed in pollen tubes ${ }^{46}$, and SphK1 and 2 are located in the same locus ${ }^{4}$, we generated an artificial miRNA-based knockdown allele targeting SphK1 and 2 in lcbk1 background. However, these transgenic lines did not show tod1-like pollen tube phenotype in pistil. This is most likely due to genetic redundancy, and there may be other genes, acting as SphK, which have not been characterized yet.

In conclusion, we demonstrated that TOD1 is a bona fide alkaline ceramidase that plays a critical role in turgor pressure regulation during pollen tube growth in vivo and in stomatal closure. This opens up an opportunity to dissect the role of sphingolipids in turgor pressure control in plant cells. Further studies on S1P signalling and sphingolipidomics will likely provide novel insights into turgor pressure regulation in plant cells.

\footnotetext{
Methods

Plant materials and growth conditions. Arabidopsis thaliana ecotypes Landsberg erecta (Ler) or Columbia (Col) were used as the wild type. tod1-2 (SALK_110396) was obtained from Arabidopsis Biological Resource Center (ABRC) ${ }^{47}$. gaut13-1 (SALK_150132) was obtained from Li-Qun Chen (China Agricultural University) $^{32}$. YC3.60 transgenic seeds were obtained from Megumi Iwano (Nara Institute of Science and Technology ${ }^{48}$. Surface sterilized seeds were germinated on Murashige and Skoog (MS) medium supplemented as required with $50 \mathrm{mgl}^{-1}$ kanamycin and/or $20 \mathrm{mgl}^{-1}$ hygromycin. The seeds were kept at $4{ }^{\circ} \mathrm{C}$ for 3 days, and then transferred to a greenhouse $\left(22^{\circ} \mathrm{C}\right)$ under long-day conditions ( $16 \mathrm{~h} \mathrm{light/}$
} day). Seedlings were transplanted to soil 10 days after plating.
Mutagenesis and screens for suppressors of tod1. Approximately 5,000 tod 1 seeds were mutagenized by imbibition of $0.3 \%$ ethyl methanesulfonate for $12 \mathrm{~h}$ at room temperature, followed by washing once with $100 \mathrm{mM} \mathrm{Na}_{2} \mathrm{~S}_{2} \mathrm{O}_{3}$ and several times with sterilized water. The $\mathrm{M}_{2}$ seeds were collected in 32 pools, each representing approximately $280 \mathrm{M}_{1}$ plants. We screened approximately $18,000 \mathrm{M}_{2}$ seeds for suppressors of tod1. Putative suppressors were backcrossed to tod 1 before further genetic and phenotypic analyses. Plants homozygous for the tod 1 allele were confirmed by PCR analysis. $\mathrm{BC}_{4} \mathrm{~F}_{2}$ of putative suppressors were crossed to tod1-2, and the resulting $\mathrm{F}_{2}$ populations were examined for the segregation of fertility. Dominance or recessiveness of putative suppressors was determined by segregation ratio of full seed set to reduced seed set. For recessive mutations, genomic DNA was isolated from individual $\mathrm{F}_{2}$ plants with full seed set. For dominant mutations, seeds from individual $\mathrm{F}_{2}$ plants with full seed set were collected and propagated separately. When all individual $F_{3}$ plants from the same $\mathrm{F}_{2}$ showed restored seed set, then genomic DNA was isolated from individual $F_{3}$ plants.

Whole-genome re-sequencing and data analysis. DNA was isolated using a DNeasy Plant Mini Kit (Qiagen). Equally pooled genomic DNA was used to prepare a library for Illumina sequencing. The library was sequenced on the HiSeq2000 platform and $100 \mathrm{bp}$ paired-end reads were generated. Reads alignment and variants identification were done according to the references with modifications ${ }^{49,50}$. Clean reads were mapped against the TAIR10 releases of the Arabidopsis genome, using the BWA software with default parameters ${ }^{51}$. Sites with more than two alleles were not considered. To eliminate bias from cross breeding, variants were removed that existed in the variant list (http://1001genomes.org/data/MPI/ MPISchneeberger2011/releases/2012_03_14/Ler-1/Marker/Ler-1.SNPs.TAIR9.txt). SNPs were further filtered with two steps: (i) extraction of SNPs that exhibit G/C-to-A/T transitions, which are the most frequent changes caused by ethyl methanesulfonate mutagenesis. (ii) SNPs with a supporting reads depth less than 20 or more than 100 were removed to reduce mapping errors. Gene mapping analysis was performed according to the reference ${ }^{51}$ with default parameters except for SNPs filtering, which indices more than 0.2 were considered. The SNP locations within the gene model were annotated using SNPEff V3.3. Genes with 'HIGH' impact SNPs were taken as candidates.

Pollen and pollen tube staining. Mature pollen grains were stained for DNA with $1 \mu \mathrm{g} \mathrm{ml}{ }^{-1}$ DAPI for $10 \mathrm{~min}$ (ref. 52), and for pollen viability with Alexander's $\operatorname{stain}^{53}$. For pollen tube staining, the pistil carpel was partially dissected using a forceps and a syringe needle, and then was fixed in acetic acid/ethanol (1:9) solution for more than $2 \mathrm{~h}$. The fixed tissue was softened in $1 \mathrm{M} \mathrm{NaOH}$ overnight, and then stained with $0.01 \%$ aniline blue in $50 \mathrm{mM}$ potassium phosphate buffer ( $\mathrm{pH}$ 7.5). The stained samples were then observed with a Zeiss Axioplan microscope (Carl Zeiss) equipped with epifluorescence.

In vitro pollen germination and pollen tube growth. Pollen grains from fully opened flowers were spread on pollen germination medium containing $5 \mathrm{mM}$ $\mathrm{CaCl}_{2}, 5 \mathrm{mM} \mathrm{KCl}, 1 \mathrm{mM} \mathrm{MgSO} 4,0.01 \% \mathrm{H}_{3} \mathrm{BO}_{3}, 10 \%$ sucrose (w/v) and $1.5 \%$ agarose (for solid medium), $\mathrm{pH} 7.5$, and incubated at $22^{\circ} \mathrm{C}$ (ref. 54). The germination rate and pollen tube lengths were determined with a Zeiss Axioplan microscope.

Sphingosine and S1P were dissolved in methanol to make stock solution. The complementation experiment were conducted in $1.5 \%$ ultra-low gelling agarose (Sigma-Aldrich) germination medium with additions of $10 \mu \mathrm{M}$ Sphingosine (860490 P, Avanti) or S1P (S9666, Sigma), or the same volume of methanol for the control.

Liquid pollen tube germination was performed in $2 \mathrm{ml}$ Eppendorf tube, using 20 flowers for each $200 \mu \mathrm{l}$ medium.

Scanning electron microscopy. Pistils pollinated with either Ler or tod 1 were partially dissected $24 \mathrm{~h}$ after pollination and immediately fixed with $3 \%$ glutaraldehyde in $100 \mathrm{mM}$ sodium phosphate buffer, $\mathrm{pH}$ 7.0. After an initial fixation for $1 \mathrm{~h}$ at room temperature under a gentle vacuum, samples were kept in fresh fixative at $4{ }^{\circ} \mathrm{C}$ overnight. Pistils were then rinsed three times in sodium phosphate buffer and dehydrated through an ethanol series. After dehydration, ethanol was replaced by isoamyl acetate. Afterwards, specimens were dried with a critical point drier using liquid carbon dioxide. The dried pistils were mounted on stubs for further dissection and sputter coating with gold. Specimens were observed with a SEM (Hitachi). For stomatal aperture observation, siliques were fixed after ABA or S1P treatment.

Molecular analysis and genetic complementation. Genomic DNA of tod 1 was extracted by CTAB method ${ }^{55}$. The flanking sequence of $D s$ element was isolated by thermal asymmetric interlaced $P_{C R}^{21}$. TOD1 genomic DNA containing its promoter sequence was amplified with primers TOD1cPF and TOD1gBR and cloned into pCAMBIA1300 at PstI and BamHI sites. Total RNA was isolated from different tissues of Ler with Trizol (Invitrogen). For first strand cDNA synthesis, $2 \mu \mathrm{g}$ of total RNA was used for reverse transcription with M-MLV reverse 
transcriptase (Promega) according to the manufacturer's instructions. RT-PCR was performed with primer pair TOD1RTF and TOD1RTR. Arabidopsis eIF4A was used as an internal control with primers $e I F 4 A F$ and $e I F 4 A R$. PCR products were checked by $1.2 \%$ agarose gel electrophoresis. Uncropped gel images are shown in Supplementary Fig. 8. Real-time RT-PCR reactions containing SYBR Green I were performed on CFX96 real-time system (Bio-Rad). Primers TOD1QF and TOD1QR were used for TOD1. Primers ACT2QF and ACT2QR for ACTIN2 were used to normalize the real-time RT-PCR data. Deletion variants 1-409 using primers TOD1c1XF and TOD1c409KR, 20-462 using primers TOD1c20XF and TOD1c462KR, and 40-462 using primers TOD1c40XF and TOD1c462KR were generated by RT-PCR, digested by $X b a \mathrm{I}$ and $K p n \mathrm{I}$ and ligated into pCAMBIA1300-Lat52::NOSter under the control of Lat52 promoter ${ }^{56}$. These constructs were introduced into plants via Agrobacterium-mediated transformation ${ }^{57}$. All primers are listed in Supplementary Table 2.

To express TOD1-eGFP under its native promoter, TOD1 genomic DNA without the $3^{\prime}$-UTR was amplified using primer pair TOD $1 \mathrm{c} P \mathrm{~F}$ and $T O D 1 \mathrm{c} B \mathrm{R}$, and cloned into pCAMBIA1300-eGFP with $3^{\prime}$-UTR of TOD1 at the carboxy terminus of eGFP (Supplementary Table 2).

Bioinformatic analysis. The TOD1 amino-acid sequence was used for BLASTP search through National Center for Biotechnology Information (http:// www.ncbi.nlm.nih.gov/) and gene families search through PANTHER (http:// www.pantherdb.org/). The phylogenetic tree of alkaline ceramidases was generated using the neighbour-joining method in MEGA4 (ref. 58). The alignment was generated by CUSTAL W with default parameters (http://npsa-pbil.ibcp.fr/cgi-bin/ npsa_automat.pl?page=/NPSA/npsa_clustalw.html) ${ }^{59}$.

\section{Construction of pTOD1:gTOD1-GUS and GUS assay. TOD1 $3^{\prime}$-UTR} was amplified using primer pair $3 U T R F$ and $3 U T R R$ and subcloned into pCAMBIA1300-GUS at the C terminus of GUS, after KpnI and EcoRI digestion. Full-length TOD1 genomic DNA containing its promoter sequence was amplified using primer pair $T O D 1 \mathrm{cPF}$ and $T O D 1 \mathrm{cBR}$ and cloned into the aforementioned binary vectors at the $\mathrm{N}$ terminus of GUS after Pst I and Bam HI digestion, yielding pTOD1::gTOD1-GUS (Supplementary Table 2). This construct was then transferred into Col as described above. TOD1::gTOD1-GUS transgenic plants were selected on MS medium containing $20 \mathrm{mg} \mathrm{l}^{-1}$ hygromycin. GUS staining was performed as described ${ }^{60}$. Mature pollen grains were spread on pollen germination medium (solid), and transferred for GUS staining after $6 \mathrm{~h}$ growth at $22^{\circ} \mathrm{C}$. Tissues were cleared in $20 \%$ lactic acid and $20 \%$ glycerol, and observed with a Zeiss Axioplan microscope.

Transient transformation of tobacco pollen grains. The full-length coding sequence of TOD1 was amplified by RT-PCR using primer pair TOD1cEF and TOD1cBR (Supplementary Table 2). The PCR product was digested with EcoRI and BamHI, and then cloned into pBSK-Lat52::mCherry. The expression vector was co-transfected with green fluorescent protein (GFP)-fused endomembrane system markers ${ }^{61}$. Tobacco pollen grains were co-transfected by bombardment ${ }^{62}$.

Imaging of pollen tubes was performed using CLSM (Zeiss LSM510 META). Signals were captured using a 488-nm excitation laser for GFP, and a 543-nm excitation laser for mCherry. Images were processed with Zeiss LSM Image Browser (Release 3.2) and Photoshop.

Expression of TOD1 in yeast. The open reading frame of TOD1 was amplified by PCR using primer pair Flag-TOD1F and Flag-TOD1R; YPC1 was amplified by PCR primers Flag-YPC1F and Flag-YPC1R (Supplementary Table 2). PCR products were digested by $K p n I$ and EcoRI and cloned into the same restriction sites of pYes2. The expression vector pYes2-Flag-TOD1 or pYes2-Flag-YPC1 was transformed into the yeast strain $\Delta y p c 1 \Delta y d c 1$ using lithium acetate ${ }^{63}$. The yeast double knockout strain $\Delta y p c 1 \Delta y d c 1$ lacking endogenous yeast ceramidases YPC1p and YDC1p was provided by Cungui Mao (Medical University of South Carolina) ${ }^{28}$. The strain containing pYes2-Flag-TOD1 was maintained in synthetic dropout (SD) medium (SD/-Ura) with glucose (Clontech). The Flag-tagged TOD1 was induced by $2 \%$ galactose in $\mathrm{SD} /$-Ura medium with raffinose.

Microsome preparation and immunoblotting. Yeast cells were suspended in lysis buffer ( $25 \mathrm{mM}$ Tris/HCl, pH 7.4, containing $1 \mathrm{mM}$ phenylmethyl sulphonyl fluoride and cocktail) after being washed twice with ice-cold double-distilled deionized water. Cells were homogenized ten times for $40 \mathrm{~s}$ with $1 \mathrm{~min}$ chilling on ice between each homogenization with acid-washed glass beads (Sigma) using a TissueLyser (Qiagen) set at the maximum speed. The supernatant was transferred to a new Eppendorf tube after centrifugation at $700 \mathrm{~g}$ for $5 \mathrm{~min}$ at $4{ }^{\circ} \mathrm{C}$. Cell debris was removed by centrifugation at $1,400 \mathrm{~g}$ for $5 \mathrm{~min}$ at $4{ }^{\circ} \mathrm{C}$. To pellet the membrane fraction, the supernatant was centrifuged at 40,000 r.p.m. for $1 \mathrm{~h}$ at $4{ }^{\circ} \mathrm{C}$ in a Beckman Optimal L-100XP ultracentrifuge. The membrane fraction was rinsed gently with the lysis buffer and resuspended in lysis buffer. The protein concentration was determined by BCA Protein Assay Kit (TianGen). For immunoblotting, about $20 \mu \mathrm{g}$ proteins was separated on a $10 \%$ SDS-polyacrylamide gel electrophoresis and transferred to a polyvinylidene difluoride membrane
(Bio-Rad), followed by immunoblotting with 1:4,000 diluted mouse anti-Flag M2 antibody (F3165, Sigma-Aldrich) and 1:10,000 diluted goat anti-mouse IgG-HRP secondary antibody (CW0102, Beijing CoWin Biotech). The uncropped blot image is shown in Supplementary Fig. 8.

Ceramidase activity assay. Ceramidase activity was assayed using fluorescent NBD-C 12 -phytoceramide (810215 P, Avanti), and NBD-C ${ }_{12}$-ceramide $(810211 \mathrm{P}$ Avanti) as substrates. The substrate was dissolved in reaction buffers with different $\mathrm{pHs}^{64}$. In each reaction, $8 \mathrm{nM}$ substrate in $20 \mu \mathrm{l}$ reaction buffer was added to $20 \mu \mathrm{l}$ of yeast microsomes ( $50 \mu \mathrm{g}$ protein) in lysis buffer. $\mathrm{CaCl}_{2}$ was added to a final concentration of $5 \mathrm{mM}$. The reaction was performed at $37^{\circ} \mathrm{C}$ for $90 \mathrm{~min}$, and stopped by addition of methanol/chloroform (1:1). The mixture was vortexed for $30 \mathrm{~s}$, kept at room temperature for $5 \mathrm{~min}$ and then centrifuged. The organic phase was collected and dried. Lipids were dissolved in $20 \mu \mathrm{l} \mathrm{methanol/chloroform} \mathrm{(1:2)}$ then spotted on TLC plates (Merck). The substrate and product were separated by developing the plates in chloroform $/$ methanol $/ 25 \%$ ammonium hydroxide (90:30:0.5) solvent system. The NBD-fatty acid product was identified by comparison with C12-NBD-fatty acid (72963, Sigma) standard. The TLC plates were scanned using PhosphorImager (Typhoon Trio) system set at fluorescence mode. Uncropped TLC images are shown in Supplementary Fig. 8.

Stomatal bioassays. Promotion of stomatal closure by ABA was performed with siliques. Siliques were floated in $\mathrm{MES} / \mathrm{KCl}$ buffer $(5 \mathrm{mM} \mathrm{KCl}, 10 \mathrm{mM} \mathrm{MES}$ and $50 \mu \mathrm{M} \mathrm{CaCl}_{2}, \mathrm{pH}$ 6.15) under illumination for $2.5 \mathrm{~h}$ (ref. 42), then mock control or $10 \mu \mathrm{M}$ ABA was added, and siliques incubated for another $2 \mathrm{~h}$, then fixed for SEM observation. S1P-induced promotion of stomatal closure was conducted in a similar way. The aperture ratio (width/length) was calculated.

Turgor pressure measurement. The turgor pressure of pollen tubes was measured by incipient plasmolysis method as described ${ }^{8}$, with modifications. Pollen tubes after $5 \mathrm{~h}$ germination were pipetted into glass bottom dishes. An aliquot $(60 \mu \mathrm{l})$ of original growth medium was collected for osmolality measurement denoting $\left(\pi_{\mathrm{e}}\right)_{0}$. Pollen tubes were observed under CLSM. Liquid pollen germination medium with $10 \%$ sucrose and $10 \%$ mannitol was added into the growth medium until the plasma membrane retracted from the cell wall of the tube tip. An aliquot of $60 \mu \mathrm{l}$ of solution was collected for osmolality measurement denoting $\pi_{\mathrm{i}}$. The osmolality was measured by Fiske Micro-Osmometer. Turgor pressure is given by $\pi_{\mathrm{i}}-\left(\pi_{\mathrm{e}}\right)_{0}$.

$\mathrm{Ca}^{2}+$ imaging of pollen tubes. To generate YC3.60-expressing tod 1 mutants, tod1-2 was crossed with YC3.60-expressing $\mathrm{Col}^{48}$. The resulting $\mathrm{F}_{1}$ progeny was allowed to self-pollinate with limited pollen grains. The genotype of individual $\mathrm{F}_{2}$ plants was determined by PCR. Pollen grains from YC3.60-expressing tod1-2 or $\mathrm{Col}$ were germinated on agarose medium. Imaging of growing pollen tubes was performed using CLSM with a 458-nm argon laser. Emission was measured at 470-500 for ECFP and 515-550 for Venus. Venus/ECFP ratio images were processed by Image $\mathrm{J}$ and Image-pro Plus.

\section{References}

1. Ishijima, A., Doi, T., Sakurada, K. \& Yanagida, T. Sub-piconewton force fluctuations of actomyosin in vitro. Nature 352, 301-306 (1991).

2. Suter, D. M. \& Miller, K. E. The emerging role of forces in axonal elongation. Prog. Neurobiol. 94, 91-101 (2011)

3. Lew, R. R. How does a hypha grow? The biophysics of pressurized growth in fungi. Nat. Rev. Microbiol. 9, 509-518 (2011).

4. Schopfer, P. Biomechanics of plant growth. Am. J. Bot. 93, 1415-1425 (2006)

5. Proseus, T. E. \& Boyer, J. S. Periplasm turgor pressure controls wall deposition and assembly in growing Chara corallina cells. Ann. Bot. 98, 93-105 (2006).

6. Hiscock, S. J. \& Allen, A. M. Diverse cell signalling pathways regulate pollenstigma interactions: the search for consensus. New Phytol. 179, 286-317 (2008)

7. Berry, J. A., Beerling, D. J. \& Franks, P. J. Stomata: key players in the earth system, past and present. Curr. Opin. Plant. Biol. 13, 233-240 (2010).

8. Benkert, R., Obermeyer, G. \& Bentrup, F. W. The turgor pressure of growing lily pollen tubes. Protoplasma 198, 1-8 (1997).

9. Zerzour, R., Kroeger, J. \& Geitmann, A. Polar growth in pollen tubes is associated with spatially confined dynamic changes in cell mechanical properties. Dev. Biol. 334, 437-446 (2009).

10. Kroeger, J. H., Zerzour, R. \& Geitmann, A. Regulator or driving force? The role of turgor pressure in oscillatory plant cell growth. PLoS One 6, e18549 (2011)

11. Taylor, L. P. \& Hepler, P. K. Pollen germination and tube growth. Annu. Rev Plant Physiol. Plant Mol. Biol. 48, 461-491 (1997).

12. Lord, E. M. \& Russell, S. D. The mechanisms of pollination and fertilization in plants. Annu. Rev. Cell Dev. Biol. 18, 81-105 (2002).

13. Crawford, B. C. W., Ditta, G. \& Yanofsky, M. F. The NTT gene is required for transmitting-tract development in carpels of Arabidopsis thaliana. Curr. Biol. 17, 1101-1108 (2007). 
14. Berger, F., Hamamura, Y., Ingouff, M. \& Higashiyama, T. Double fertilization caught in the act. Trends Plant Sci. 13, 437-443 (2008).

15. Hannun, Y. A. \& Obeid, L. M. Principles of bioactive lipid signalling: lessons from sphingolipids. Nat. Rev. Mol. Cell Biol. 9, 139-150 (2008).

16. Mao, C. G. et al. Cloning and characterization of a novel human alkaline ceramidase - A mammalian enzyme that hydrolyzes phytoceramide. J. Biol. Chem. 276, 26577-26588 (2001)

17. Pata, M. O. et al. Molecular cloning and characterization of OsCDase, a ceramidase enzyme from rice. Plant J. 55, 1000-1009 (2008).

18. Sundaresan, V. et al. Patterns of gene action in plant development revealed by enhancer trap and gene trap transposable elements. Genes Dev. 9, 1797-1810 (1995).

19. Page, D. R. \& Grossniklaus, U. The art and design of genetic screens: Arabidopsis thaliana. Nat. Rev. Genet. 3, 124-136 (2002).

20. Johnson-Brousseau, S. A. \& McCormick, S. A compendium of methods useful for characterizing Arabidopsis pollen mutants and gametophytically-expressed genes. Plant J. 39, 761-775 (2004).

21. Liu, Y. G. et al. Efficient isolation and mapping of Arabidopsis thaliana T-DNA insert junctions by thermal asymmetric interlaced PCR. Plant J. 8, 457-463 (1995).

22. Mi, H. et al. The PANTHER database of protein families, subfamilies, functions and pathways. Nucleic Acids Res. 33, D284-D288 (2005).

23. Thomas, P. D. et al. PANTHER: a library of protein families and subfamilies indexed by function. Genome Res. 13, 2129-2141 (2003).

24. Mao, C. G. et al. Cloning and characterization of a mouse endoplasmic reticulum alkaline ceramidase - An enzyme that preferentially regulates metabolism of very long chain ceramides. J. Biol. Chem. 278, 31184-31191 (2003).

25. Xu, R. J. et al. Golgi alkaline ceramidase regulates cell proliferation and survival by controlling levels of sphingosine and S1P. FASEB J 20, 1813-1825 (2006).

26. Sun, W. et al. Upregulation of the human alkaline ceramidase 1 and acid ceramidase mediates calcium-induced differentiation of epidermal keratinocytes. J. Invest. Dermatol. 128, 389-397 (2008).

27. Mao, C. G., Xu, R. J., Bielawska, A. \& Obeid, L. M. Cloning of an alkaline ceramidase from Saccharomyces cerevisiae - An enzyme with reverse (CoA-independent) ceramide synthase activity. J. Biol. Chem. 275, 6876-6884 (2000).

28. Mao, C. G., Xu, R. J., Bielawska, A., Szulc, Z. M. \& Obeid, L. M. Cloning and characterization of a Saccharomyces cerevisiae alkaline ceramidase with specificity for dihydroceramide. J. Biol. Chem. 275, 31369-31378 (2000).

29. Mao, Z. H. et al. Alkaline ceramidase 2 (ACER2) and its product dihydrosphingosine mediate the cytotoxicity of $\mathrm{N}$-(4-hydroxyphenyl) retinamide in tumor cells. J. Biol. Chem. 285, 29078-29090 (2010).

30. Coursol, S. et al. Sphingolipid signalling in Arabidopsis guard cells involves heterotrimeric G proteins. Nature 423, 651-654 (2003).

31. Ng, C. K. Y., Carr, K., McAinsh, M. R., Powell, B. \& Hetherington, A. M. Drought-induced guard cell signal transduction involves sphingosine-1phosphate. Nature 410, 596-599 (2001).

32. Wang, L. et al. Arabidopsis galacturonosyltransferase (GAUT) 13 and GAUT14 have redundant functions in pollen tube growth. Mol. Plant 6, 1131-1148 (2013).

33. Zonia, L. \& Munnik, T. Life under pressure: hydrostatic pressure in cell growth and function. Trends Plant Sci. 12, 90-97 (2007).

34. Winship, L. J., Obermeyer, G., Geitmann, A. \& Hepler, P. K. Under pressure, cell walls set the pace. Trends Plant Sci. 15, 363-369 (2010).

35. Zonia, L. \& Munnik, T. Understanding pollen tube growth: the hydrodynamic model versus the cell wall model. Trends Plant Sci. 16, 347-352 (2011).

36. Winship, L. J., Obermeyer, G., Geitmann, A. \& Hepler, P. K. Pollen tubes and the physical world. Trends Plant Sci. 16, 353-355 (2011).

37. Parre, E. \& Geitmann, A. Pectin and the role of the physical properties of the cell wall in pollen tube growth of Solanum chacoense. Planta 220, 582-592 (2005).

38. Bosch, M. \& Hepler, P. K. Silencing of the tobacco pollen pectin methylesterase NtPPME1 results in retarded in vivo pollen tube growth. Planta 223, 736-745 (2005).

39. Wu, M. M. et al. Organelle $\mathrm{pH}$ studies using targeted avidin and fluoresceinbiotin. Chem. Biol. 7, 197-209 (2000).

40. Nakamura, N., Tanaka, S., Teko, Y., Mitsui, K. \& Kanazawa, H. Four $\mathrm{Na}^{+} / \mathrm{H}^{+}$ exchanger isoforms are distributed to Golgi and post-Golgi compartments and are involved in organelle pH regulation. J. Biol. Chem. 280, 1561-1572 (2005).

41. Markham, J. E. \& Jaworski, J. G. Rapid measurement of sphingolipids from Arabidopsis thaliana by reversed-phase high-performance liquid chromatography coupled to electrospray ionization tandem mass spectrometry. Rapid Commun. Mass Spectrom 21, 1304-1314 (2007).

42. Michaelson, L. V. et al. Functional characterization of a higher plant sphingolipid $\Delta 4$-desaturase: defining the role of sphingosine and sphingosine-1-phosphate in Arabidopsis. Plant Physiol. 149, 487-498 (2009).
43. Worrall, D. et al. Involvement of sphingosine kinase in plant cell signalling. Plant J. 56, 64-72 (2008).

44. Guo, L., Mishra, G., Taylor, K. \& Wang, X. M. Phosphatidic acid binds and stimulates Arabidopsis sphingosine kinases. J. Biol. Chem. 286, 13336-13345 (2011).

45. Imai, H. \& Nishiura, H. Phosphorylation of sphingoid long-chain bases in Arabidopsis: Functional characterization and expression of the first sphingoid long-chain base kinase gene in plants. Plant Cell Physiol. 46, 375-380 (2005).

46. Qin, Y. et al. Penetration of the stigma and style elicits a novel transcriptome in pollen tubes, pointing to genes critical for growth in a pistil. PLoS Genet. 5, e1000621 (2009).

47. Alonso, J. M. et al. Genome-wide insertional mutagenesis of Arabidopsis thaliana. Science 301, 653-657 (2003).

48. Iwano, M. et al. Fine-tuning of the cytoplasmic $\mathrm{Ca}^{2+}$ concentration is essential for pollen tube growth. Plant Physiol. 150, 1322-1334 (2009).

49. The 3,000 Rice Genomes Project. The 3,000 rice genomes project. Gigascience 3, 7 (2014).

50. Abe, A. et al. Genome sequencing reveals agronomically important loci in rice using MutMap. Nature Biotechnol. 30, 174-178 (2012).

51. Li, H. \& Durbin, R. Fast and accurate short read alignment with Burrows-Wheeler transform. Bioinformatics 25, 1754-1760 (2009).

52. Regan, S. M. \& Moffatt, B. A. Cytochemical analysis of pollen development in wild-type Arabidopsis and a male-sterile mutant. Plant Cell 2, 877-889 (1990).

53. Alexander, M. P. A versatile stain for pollen fungi, yeast and bacteria. Stain. Technol 55, 13-18 (1980).

54. Boavida, L. C. \& McCormick, S. Temperature as a determinant factor for increased and reproducible in vitro pollen germination in Arabidopsis thaliana. Plant J. 52, 570-582 (2007).

55. Rogers, S. O. \& Bendich, A. J. Extraction of DNA from milligram amounts of fresh, herbarium and mummified plant-tissues. Plant Mol. Biol. 5, 69-76 (1985).

56. Twell, D., Wing, R., Yamaguchi, J. \& Mccormick, S. Isolation and expression of an anther-specific gene from tomato. Mol. Gen. Genet. 217, 240-245 (1989).

57. Clough, S. J. \& Bent, A. F. Floral dip: a simplified method for Agrobacteriummediated transformation of Arabidopsis thaliana. Plant J. 16, 735-743 (1998).

58. Tamura, K., Dudley, J., Nei, M. \& Kumar, S. MEGA4: Molecular evolutionary genetics analysis (MEGA) software version 4.0. Mol. Biol. Evol. 24, 1596-1599 (2007).

59. Combet, C., Blanchet, C., Geourjon, C. \& Deléage, G. NPS@: Network Protein Sequence Analysis. Trends Biochem. Sci. 25, 147-150 (2000).

60. Vielle-Calzada, J. P., Baskar, R. \& Grossniklaus, U. Delayed activation of the paternal genome during seed development. Nature 404, 91-94 (2000).

61. Cheung, A. Y. et al. Rab2 GTPase regulates vesicle trafficking between the endoplasmic reticulum and the Golgi bodies and is important to pollen tube growth. Plant Cell 14, 945-962 (2002).

62. Wang, H. \& Jiang, L. W. Transient expression and analysis of fluorescent reporter proteins in plant pollen tubes. Nat. Protoc. 6, 419-426 (2011).

63. Gietz, R. D. \& Schiestl, R. H. Quick and easy yeast transformation using the LiAc/SS carrier DNA/PEG method. Nat. Protoc. 2, 35-37 (2007).

64. Galadari, S. et al. Identification of a novel amidase motif in neutral ceramidase. Biochem. J. 393, 687-695 (2006).

\section{Acknowledgements}

We thank Megumi Iwano for YC3.60 transgenic seeds, Li-Qun Chen for gaut13-1 mutant seeds, Cungui Mao for yeast mutant $\Delta y p c 1 \Delta y d c 1$ and Alice Cheung (University of Massachusetts) for GFP-Rab2 plasmid. We appreciate the expertise of Chengzhi Liang and Haofeng Chen (Chinese Academy of Sciences) in whole-genome re-sequencing and analysis. We are grateful to Sheila McCormick, Michael R. Schläppi and Megan E. Griffith for critical reading of the manuscript. This work was supported by grants from the Ministry of Science and Technology of China (2013CB945103) and the National Natural Science Foundation of China (30830063) to W.-C.Y. and (31370345) to D.-Q.S.

\section{Author contributions}

L.-Y.C., W.-J.Z. and W.-C.Y. designed the research; L.-Y.C., W.-J.Z. and D.-Q.S performed the research; Z.-S.T. and J.L. contributed new reagents/analytic tools; L.-Y.C., D.-Q.S., W.-J.Z. and W.-C.Y. analysed the data; L.-Y.C. and W.-C.Y. wrote the paper.

\section{Additional information}

Accession codes: Illumina sequencing data associated with this study have been submitted to the National Center for Biotechnology Information Sequence Read Archive under accession code SRR1652473.

Supplementary Information accompanies this paper at http://www.nature.com/ naturecommunications

Competing financial interests: The authors declare no competing financial interests. 
Reprints and permission information is available online at http://npg.nature.com/ reprintsandpermissions/

How to cite this article: Chen, L.-Y. et al. The Arabidopsis alkaline ceramidase TOD1 is a key turgor pressure regulator in plant cells. Nat. Commun. 6:6030 doi: $10.1038 /$ ncomms7030 (2015). (c) (i) This work is licensed under a Creative Commons Attribution 4.0 International License. The images or other third party material in this article are included in the article's Creative Commons license, unless indicated otherwise in the credit line; if the material is not included under the Creative Commons license, users will need to obtain permission from the license holder to reproduce the material. To view a copy of this license, visit http://creativecommons.org/licenses/by/4.0/ 